Journal of Computer Science 8 (1): 170-174, 2012

ISSN 1549-3636

(C) 2012 Science Publications

\title{
A Review on Recent Research and Implementation Methodologies on Medical Image Segmentation
}

\author{
${ }^{1}$ S.K. Somasundaram and ${ }^{2}$ P. Alli \\ ${ }^{1}$ Department of CSE, PSNA College of Engineering and Technology, \\ Dindigul, Tamil Nadu, India \\ ${ }^{2}$ Velammal College of Engineering and Technology, \\ Madurai, Tamil Nadu, India
}

\begin{abstract}
Problem statement: Image segmentation plays a very important role in digital imaging applications for classifying the anatomical structures and other Regions of Interest. Segmentation refers to the process of identifying and isolating the surface and regions of the digital image which corresponds to the structural units. Approach: In the past few decades, many methods have been proposed to segment the digital image. Most of them were based on two basic properties of the pixels in relation to their local neighborhood: discontinuity and similarity. The approaches based on discontinuity partition of an image are performed by detecting isolated points, lines and edges, which were known as edge detection techniques. Results: A particular class of segmentation techniques is based on multi resolution analysis. The image segmentation process is includes various techniques like Threshold method, boundary tracking, Clustering, Region-Based. Conclusion: The objective of this study is to provide brief study on various segmentation methodologies for the researchers. This study presents the brief review on image segmentation and analyses the performance and improvement of various algorithms and fulfilled the objectives.
\end{abstract}

Key words: Image processing, Pulse-Coupled Neural Network (PCNN), Feed Forward (FF), various algorithms, computer vision methods, transformed methods, Back-Propagation (BP)

\section{INTRODUCTION}

Image processing is a major area of concern mainly due to the availability of bulk amount of sensitive and vital images. Its development has been increased by technological advancement in Digital Imaging, Computer Architectures, Computer Processors and mass Storage devices. Fields that used analog imaging are now switching to digital systems, for their flexibility and effectiveness. Image Processing can be used in the fields of medicine, film and video production, photography, remote sensing, security monitoring. Image processing processes the image (input) from various sources to either an image (output) or, a set of parameters or characteristics related to the image. The primary objective of Digital image processing is extracting useful information from images without human assistance. Segmentation is the most essential task of image processing for processing satellite image, Medical images. The other image processing techniques are image reduction, extraction and enhancement. When the input data is too huge to be processed or often repetitive, then the input data will be transformed into a reduced representation set of features. Transforming the input data into the set of features is called feature extraction. Fast image segmentation method can be more useful and valuable in the field of Medical imaging, Satellite imaging and underwater imaging. Till date there are several general purpose algorithms and techniques were developed for image segmentation.

The image segmentation is a first step in many computer vision methods that groups the pixels of the image in logical ways. Since, there are many levels of detail in an image and many possible ways of meaningfully grouping pixels, it is a cumbersome task to define Image segmentation.

The objective of the image segmentation is separating a given input image into different regions or edges with homogenous properties as in Fig. 1 (a) and (b). In texture segmentation algorithm of color image is assigning a label to each pixel based on its color properties and texture properties. There are variety of significant study have focused on developing algorithms based on either color, grey scale or texture features separately. The actual image gathered in nature generally contains the combination of color, texture and/or pattern.

Corresponding Author: S.K. Somasundaram, Department of CSE, PSNA College of Engineering and Technology, Indigo, Tamil Nadu, India 


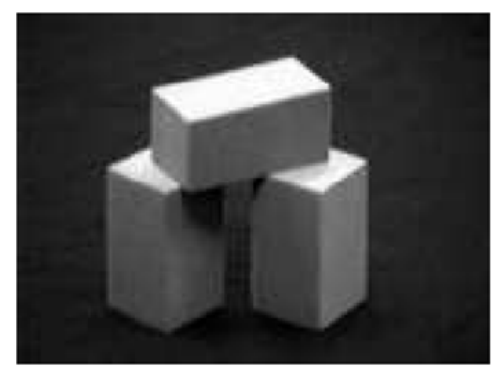

(a)

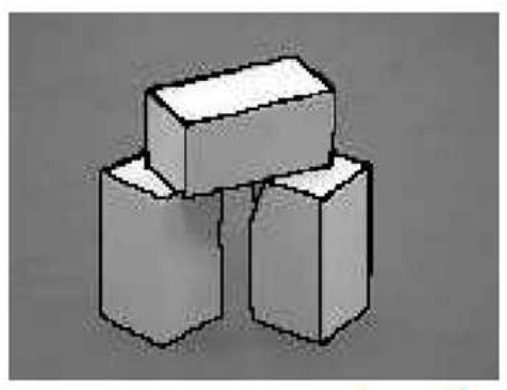

(b)

Fig.1: Image segmentation Original Image Segmentation process as indicated by dark black lines

Therefore, combining color as well as texture features are the significant benefit in distinguishing regions having the same color but different textures and vice versa. In the past few decades, many methods have been proposed to segment the color image. Most of them were based on two basic properties of the pixels in relation to their local neighborhood: discontinuity and similarity (Joes et al., 2004). The discontinuity partition of an image detects isolated points, lines and edges, which were known as edge detection techniques. A particular class of segmentation techniques ( $\mathrm{Li}$ and Chutatape, 2004) is based on multi resolution analysis. Such methods are typically based on multi resolution transformations. In general, small details are detected in higher resolution images, while larger objects are segmented in coarser images (low resolution images).

\section{MATERIALS AND METHODS}

Segmentation methods in general can be classified into three classes such as Pixel-based methods, Regional methods and Edge based methods. Pixelbased methods are the simplest of all the above methods and easiest technique to understand and implement. But these methods are not affordable nowadays because it is the least powerful and operates on one element at time, which may lead to noise. Few segmentation methods are discussed below:

Pixel-based methods: The pixel based method is a straightforward method, which applies threshold technique. Threshold method is applied for converting an intensity image into a binary image or grayscale image which normally identifying the region faster than other traditional method. The threshold technique applies a minimum and maximum threshold values for intensity of the pixels and to identify upper and lower boundaries.

The simplest definition of the pixel based method is termed as the selection of pixels which have a gray value between two given borders. The borders of the images are $g_{\min }$ and $g_{\max }$ and at least one of the borders can be infinity. Then the result $\mathrm{I}_{\text {thres }}$ after applying the threshold method to an image I with borders $\mathrm{g}_{\min }$ and $\mathrm{g}_{\max }$ are given in Eq. 1:

$$
\mathrm{I}_{\text {thres }}(\mathrm{x}, \mathrm{y})=\left\{\begin{array}{c}
1 \text { if } \mathrm{g}_{\min } \leq \mathrm{I}(\mathrm{x}, \mathrm{y}) \leq \mathrm{g}_{\max } \\
0 \text { otherwise }
\end{array}\right.
$$

This threshold method is efficient for black and white image segmentation and grey scale image segmentation. The black images and grey scale images are represented in the one dimensional, whereas the color images are represented in multi dimensional. Color space is a geometric space that can represent color perception at different point of views which usually composed by 3 or 4 attributes based on the methods. Different attributes of different color spaces are derived by different mathematics for each special purpose. Eighteen color spaces including ATD, CMY, I1 I2 I3, I1' I2' I3', HSL, HIS, HSV, CIE (L*a*b*), CIE (L*u*v*), CIE $\left(\mathrm{L}^{*} \mathrm{C}^{*} \mathrm{H}_{\mathrm{ab}} *\right)$, CIE RGB, CIE XYZ, UVW, YCC, YIQ, YUV, YCbCr and YES are used everywhere for different applications. For example, the RGB color space is used for TV display and is based on the Trichromatic theory. And this RGB is used for computer images and digital printing. The other major color representation techniques are CYBK and HSB. Therefore, the color image segmentation algorithm in different color spaces lead to different segmented results, which could be used for different segmentation purposes.

\section{There are three phases in the color images segmentation:}

Phase I: Morphological methods are applied to remove the noises away from images which applied to smooth some spots on uniformed patterns. This method is explained using the opening operation which is applied on each channel (Red, Green and Blue) of color images. The opening operation is defined in the following Eq. 2: 
$\mathrm{A} \circ \mathrm{B}=(\mathrm{A} \odot \mathrm{B}) \oplus \mathrm{B}$

Where:

A $=$ Each color channel intensity

$\mathrm{B}=$ Structuring element

$\Theta=$ Erosion operation

$\oplus=$ Dilation operation

$\circ$ = Opening operation

The opening operation generally smoothes the contour of an object in images by eliminating small islands and shapes. A structuring element which also termed as mask, which is a $3 \times 3$ square shape similar to the Fig. 2.

Phase 2: Color space transformed methods are used to transform other color space to RGB. The average intracluster distance based method is a traditional method applied for transformation. In which the intra-cluster is calculated as in Eq. 3:

$\operatorname{intra}_{\mathrm{k}}=\frac{1}{\mathrm{~N}} \sum_{\mathrm{i}=1}^{\mathrm{k}} \sum_{(\mathrm{X}, \mathrm{Y}, \mathrm{Z}) \in \mathrm{c}_{\mathrm{i}}} \sqrt{\left(\mathrm{X}-\mathrm{X}_{\mathrm{i}}\right)^{2}+\left(\mathrm{Y}-\mathrm{Y}_{\mathrm{i}}\right)^{2}+\left(\mathrm{Z}-\mathrm{Z}_{\mathrm{i}}\right)^{2}}$

Where:

$\mathrm{N} \quad=$ Total number of pixels in the specific color space

$\mathrm{K}=$ Number of clusters of the image

$\mathrm{X}, \mathrm{Y}$ and $\mathrm{Z}=$ Pixels in $\mathrm{Ci}$ which is the $i$ th cluster having cluster centre $(\mathrm{Xi}, \mathrm{Yi}, \mathrm{Zi})$ calculated as the mean value of pixels in $\mathrm{Ci}$

Phase 3: Applying clustering algorithm like K-means algorithm for finding the appropriate cluster numbers and segment images in different color spaces. The cluster with the maximum average variance is split into new clusters. The variances of three color components of such a cluster $C_{i}$ is described in the Eq. 4:

$$
\begin{aligned}
& \operatorname{Var}_{X i}=\frac{1}{N_{i}} \sum_{x \in C i}\left(X-X_{i}\right)^{2} \\
& \operatorname{Var}_{Y i}=\frac{1}{N_{i}} \sum_{x \in C i}\left(Y-Y_{i}\right)^{2} \\
& \operatorname{Var}_{Z i}=\frac{1}{N_{i}} \sum_{x \in C i}\left(Z-Z_{i}\right)^{2}
\end{aligned}
$$

where, $\mathrm{Ni}$ is the number of pixels in cluster $\mathrm{Ci}$ and the average variance is $\left(\operatorname{Var}_{\mathrm{xi}}+\operatorname{Var}_{\mathrm{xi}}+\operatorname{Var}_{\mathrm{yi}}+\operatorname{Var}_{\mathrm{zi}}\right) / 3$. When a cluster with the maximum average variance is found, the cluster is divided into two new clusters $\left(\mathrm{C}_{\mathrm{i}}{ }^{\prime}\right.$ and $\left.\mathrm{C}_{\mathrm{i}}{ }^{\prime}{ }^{\prime}\right)$ calculated as in Eq. 5:

\begin{tabular}{|l|l|l|}
\hline 1 & 1 & 1 \\
\hline 1 & 1 & 1 \\
\hline 1 & 1 & 1 \\
\hline
\end{tabular}

Fig. 2: An example of $3 \times 3$ structuring element

$$
\begin{aligned}
& C_{i}{ }^{\prime}=\left(X_{i}-X_{a}, Y_{i}-Y_{a}, Z_{i}-Z_{a}\right) \\
& C_{i}{ }^{\prime}=\left(X_{i}+X_{a}, Y_{i}+Y_{a}, Z_{i}+Z_{a}\right)
\end{aligned}
$$

In general, the pixel-based approaches can lead to serious errors due to noise.

Multi-scale segmentation: In multi scale Image segmentation, the segmentation processes are carried out in multiple scales in the scale-space. In addition to these processes, it is sometime redefined from coarse level to fine level of large scales. A common requirement in the multilevel segmentation is each and every region of the images is connected in some technical value. Witkin (1983) proposes a seminal study which includes the notion of one-dimensional signal is clearly segmented into regions, with one scale parameter for controlling the scale of segmentation. In this method, the author defined the zero-crossings of the second derivatives, such as the basic function of minima and maxima in the first derivative or the slope function of multi-scale-smoothed method of a signal. This study also redefines the hierarchical relationship between segments at different scales and this This Witkin method is specific only for one-dimensional signals and this method not a trivial method for transferring to higher-dimensional images.

Lifshitz and Pizer (1990) propose a method of study, in which the author explains the evolving procedure of iso-intensity contours over large scales and then analyzed the multi-scale method in more detailed representation. Gauch and Pizer (1993) studied the problem of multi-scale image segmentation and focused for ridges and valleys at multiple scales. A hyper stack method proposes by Vincken et al. (1997) and Tabb and Ahuja (1997) are redefining the probabilistic relations between the image structures at different scales. In the recent study, multi-scale image segmentation method is linking image structures over large scales are selected by Florack and Kuijper (2000).

Artificial Neural Networks (ANN) based segmentation: ANN is used for variety of classification and prediction such as market analysis, prediction of electrical tariff. ANN is a field of study, in which the 
artificial intelligence using the intelligence learned from the human neuron system and its decision making behavior is applied. The ANN segmentation uses the processing of small areas of an image in the small neurons (Pathegama and Gol, 2005) or a set of ANN. The cat's visual cortex model based Pulse-Coupled Neural Network (PCNN) is proposed in the past few years for segmentation. This method is developed for segmenting the high-performance biometric images. Each neuron in this PCNN corresponds to one pixel in the given input image, which receives its corresponding pixel's color information (e.g., intensity) as an external stimulus. Mohan and Baskaran (2010; 2011a, 2011b, 2011c, 2011d, 2011e; Mohan et al., 2008) proposed ANN for decision making and prediction model for network routing.

\section{RESULTS}

This study reviews the past approaches about various segmentation approaches and the limitations of the approaches are discussed. The performance of pixel based methods is improved by combining other methodologies but using threshold method alone is not possible to completely separate the regions of the images.

In the ANN based segmentation, there are various methods are applied which includes Back-Propagation (BP) method, Kohonen method and Feed Forward (FF) ANN. The study compares the various ANN methodologies and suggested recent study like PCNN.

\section{DISCUSSION}

A Multi-scale segmentation (Palenichka et al., 2011) of surface data using scale-adaptive region growing is proposed and the performance of this method was evaluated on LiDAR surface images. The authors proposed segmentation algorithm, which is initiated by an unsupervised learning of optimal seed positions through the surface attribute clustering with a twocriterion score function.

This proposal also avoids typical segmentation errors caused by an inappropriate choice of seed points and thresholds used in the region-growing algorithm. The scale-adaptive threshold estimate is based on the image local statistics in the neighborhoods of seed points.

A Novel color texture image segmentation algorithm based on wavelet transform and neutrosophic sets is proposed in (Sengur and Guo, 2011). In this proposed method, as the first step, the $\mathrm{L}^{*} \mathrm{u} * \mathrm{v}$ colour components and the grey scale image are obtained from the RGB colour texture input image. Then, wavelet transform is applied to grey scale image to obtain the LL, LH, HL and HH sub-bands. Only, LH and HL sub- bands are employed for further processes because most of the texture information is in the LH and HL subbands. Then the authors are calculated the mean energy using coefficients of LH and HL sub-bands in a local window. The energy is used for the characterization of texture that can be obtained by squaring the coefficients. The pixel $\mathrm{P}(\mathrm{i}, \mathrm{j})$ in the image domain for each $\mathrm{L}, \mathrm{u}$ and $\mathrm{v}$ channels and the energy component in wavelet domain is transformed into the neutron-sophic domain.

\section{CONCLUSION}

In this study, the overview of various segmentation methodologies applied for digital image processing is explained briefly. The study also reviews the research on various research methodologies applied for image segmentation and various research issues in this field of study. This study aims to provide a simple guide to the researcher for those carried out their research study in the image segmentation.

\section{REFERENCES}

Mohan, C.B. and R. Baskaran, 2010. Improving network performance by optimal load balancing using ACO based redundant link avoidance algorithm. Int. J. Comput. Sci., 7: 27-35.

Mohan, C.B. and R. Baskaran, 2011a. Reliable Transmission for Network Centric Military Networks. Euro. Jo. Scientific Res., 50: 564-574.

Mohan, C.B. and R. Baskaran, 2011b. Reliable BarrierFree Services (RBS) for heterogeneous next generation network. Adv. Power Elect. Instrument. Eng., 148: 79-82. DOI: 10.1007/978-3-642-204999_13

Mohan, C.B. and R. Baskaran, 2011c. Priority and compound rule based routing using ant colony optimization. Int. J. Hybrid Intell. Syst., 8: 93-97. DOI: $10.3233 / \mathrm{HIS}-2011-0131$

Mohan, C.B. and R. Baskaran, 2011d. A survey: Ant Colony Optimization based recent research and implementation on several engineering domain. Expert Syst. Appli., 39: 2239-2898. DOI: 10.1016/j.eswa.2011.09.076

Mohan, C.B. and R. Baskaran, 2011e. Energy aware and energy efficient routing protocol for adhoc network using restructured artificial bee colony system. High Perform. Architecture Grid Comput., 169: 473484. DOI: 10.1007/978-3-642-22577-2_65

Mohan, C.B., R. Sandeep and D. Sridharan, 2008. A data mining approach for predicting reliable path for congestion free routing using self-motivated neural network. Software Eng. Artifi. Intell. Networking Parallel/Distributed Comput., 149: 237-246. DOI: 10.1007/978-3-540-70560-4_20 
Florack, L. and A. Kuijper, 2000. The topological structure of scale-space images. J. Mathe.Imaging Vision, $\quad 12: \quad 65-79 . \quad$ DOI: 10.1023/A:1008304909717

Gauch, J.M. and S.M. Pizer, 1993. Multiresolution analysis of ridges and valleys in grey-scale images. IEEE Tran. Pattern Anal. Mach. Int., 15: 635-646. DOI: $10.1109 / 34.216734$

Joes, S., D. Michael, A. Meindert N. Max and G. Bram, 2004. Ridge-based vessel segmentation in color images of the retina. IEEE Trans. Med. Imag., 23: 501-509. DOI: 10.1109/TMI.2004.825627

$\mathrm{Li}, \mathrm{H}$. and O. Chutatape, 2004. Automated feature extraction in color retinal images by a model based approach. IEEE Trans. Biomed. Eng., 51: 246-254. DOI: 10.1109/TBME.2003.820400

Lifshitz, L.M. and S.M. Pizer, 1990. A multiresolution hierarchical approach to image segmentation based on intensity extrema. IEEE Trans. Pattern Anal. Mach. Intell., 12: 529-540. DOI: 10.1109/34.56189

Palenichka, R., A. Lakhssassi and M. Zaremba, 2011. Adaptive multi-scale segmentation of surface data using unsupervised learning of seed positions. Eng. Appli. Artifi. Intell., 24: 822-832. DOI: 10.1016/j.engappai.2011.03.004
Pathegama, M.P. and O. Gol, 2005. Edge-end pixel extraction for edge-based image segmentation. World Acad. Sci. Eng. Tech., 2: 164-167.

Sengur, A. and Y. Guo, 2011. Color texture image segmentation based on neutrosophic set and wavelet transformation. Comput. Vision Imag. Understanding, 115: 1134-1144. DOI: 10.1016/j.cviu.2011.04.001

Tabb, M. and N. Ahuja, 1997. Multiscale image segmentation by integrated edge and region detection. IEEE Tran. Imag. Proc., 6: 642-655. DOI: $10.1109 / 83.568922$

Vincken, K., A. Koster and M. Viergever, 1997. Probabilistic multiscale image segmentation. IEEE Tran. Pattern Anal. Mach. Int., 19: 109-120. DOI: 10.1109/34.574787

Witkin, A.P., 1983. Scale-space filtering: A new approach to multi-scale description. Proceedings of 84th International Joint Conference Acoustics, Speech, and Signal Processing, (IJASSSP' 83), IEEE Xploor Press, Palo Alto, pp: 150-153. DOI: 10.1109/ICASSP.1984.1172729 